\title{
Kinematic spin-fluctuation mechanism of high-temperature superconductivity
}

\author{
Nikolay M. Plakida ${ }^{1}$ and Viktor S. Oudovenko ${ }^{2}$ \\ ${ }^{1}$ Joint Institute for Nuclear Research, 141980 Dubna, Russia and \\ ${ }^{2}$ Rutgers University, New Jersey 08854, USA
}

(Dated: February 26, 2018)

\begin{abstract}
We study $d$-wave superconductivity in the extended Hubbard model in the strong correlation limit for a large intersite Coulomb repulsion $V$. We argue that in the Mott-Hubbard regime with two Hubbard subbands there emerges a new energy scale for the spin-fluctuation coupling of electrons of the order of the electronic kinetic energy $W$ much larger than the exchange energy $J$. This coupling is induced by the kinematic interaction for the Hubbard operators which results in the kinematic spinfluctuation pairing mechanism for $V \lesssim W$. The theory is based on the Mori projection technique in the equation of motion method for the Green functions in terms of the Hubbard operators. The doping dependence of superconductivity temperature $T_{c}$ is calculated for various values of $U$ and $V$.
\end{abstract}

PACS numbers: 74.20.Mn, 71.27.+a, 71.10.Fd,74.72.-h

\section{INTRODUCTION}

One of crucial issues in the superconductivity theory is to disclose the mechanism of high-temperature superconductivity (HTSC) in cuprates (see, e.g. [1, 2]). In early studies of the problem, a model of strongly correlated electrons was proposed by Anderson [3] where superconductivity occurs at finite doping in the resonating valence bond state (RVB) due to the antiferromagnetic (AF) superexchange interaction $J$. However, the intersite Coulomb interaction (CI) $V$ that in cuprates is of the order of $J$ may destroy the RVB state and superconducting pairing. Recently a competition of the intersite CI $V$ and pairing induced by the on-site CI $U$ in the Hubbard model [4] or by the intersite CI $V$ was actively discussed. In particular, in Ref. [5] it was stressed that a contribution from the repulsive well-screened weak CI in the first order strongly suppresses the pairing induced by contributions of higher orders, and a possibility for superconductivity "from repulsion" was questioned. Using the renormalization group method in Ref. [6] the extended Hubbard model with CI $V$ was studied where superconducting pairing of various symmetries, extended $s-, p$-, and $d$-wave types was found depending on the electron concentration and $V$. Following the original idea of Kohn-Luttinger [7], in Ref. [8] it was shown that the $p$ wave superconductivity exists in the electronic gas at low density with a strong repulsion $U$ and a relatively strong intersite CI $V$ (see, also 9] and references therein). Studies of the phase diagram within the extended Hubbard model in the weak correlation limit have shown that superconducting pairing of different types of symmetry, $s$, $p, d_{x y}$, and $d_{x^{2}-y^{2}}$ can occur depending on the CI between the nearest $V_{1}$ and next $V_{2}$ neighbor sites and electron hopping parameters between distant sites in a broad region of electron concentration [10].

However, in these investigations the Fermi-liquid model in the weak correlation limit, $U \lesssim W$, was considered, while cuprates are the Mott-Hubbard (more accurately, charge-transfer) doped insulators where a theory of strongly correlated electronic systems should be ap- plied for $U \gtrsim W$. Here $W \sim 4 t$ is the electronic kinetic energy for the two-dimensional Hubbard model with the nearest neighbor hopping parameter $t$. In the limit of strong correlations various numerical methods for finite clusters are commonly used. There are many investigations of the conventional Hubbard model (see, e.g. 1114]) but only few studies of the extended Hubbard model in which the intersite CI $V$ is taken into account. In particular, in Refs. 15 17] the extended Hubbard model was considered in a broad region of $U$ and $V$. The results of Refs. [15, 16] show that a strong on-site repulsion $U$ effectively enhances the $d$-wave pairing which is preserved for large values of $V \gg J$. In Ref. [17] using the slaveboson representation it was found that superconductivity is destroyed at a small value of $V=J$. We discuss these results in more detail in Sec. IVC by comparing them with our findings.

In our recent paper [18] we studied the extended Hubbard model in the limit of strong correlations by taking into account the CI $V$ and electron-phonon coupling. It was found that $d$-wave pairing with high- $T_{c}$ is mediated by the strong kinematic interaction of electrons with spin fluctuations. Contributions coming from weak CI $V$ and phonons turned out to be small since only $l=2$ harmonics of the interactions give a contribution to the $d$-wave pairing.

In the this paper, we consider superconductivity in the two-dimensional extended Hubbard model with a large intersite Coulomb repulsion $V$ in the limit of strong correlations to elucidate the spin-fluctuation mechanism of high-temperature superconductivity. We argue that in the two-subband regime for the Hubbard model for $U \gtrsim 6 t$ a spin-electron kinematic interaction is evolved from complicated commutation relations for the Hubbard operators (HOs) 19]. This interaction brings about the weak exchange interaction $J=4 t^{2} / U$ due to interband hopping and at the same time intraband hopping results in a much stronger kinematic interaction $g_{s f} \sim W \gg J$ of electrons with spin excitations. Therefore, the exchange interaction $J$ is not so important for the spinfluctuation pairing driven by the strong kinematic inter- 
action $g_{s f}$. We calculate the doping dependence of superconducting $T_{c}$ for various values of $U$ and $V$ and show that as long as $V$ does not exceed the kinematic interaction, $V \lesssim W$, the $d$-wave pairing is preserved. In calculations we use the Mori-type projection technique [20] in the equation of motion method for thermodynamic Green functions (GFs) [21] expressed in terms of the HOs. The self-energy in the Dyson equation is calculated in the selfconsistent Born approximation (SCBA) as in our previous publications [18, 22].

In Sec. II the two-subband extended Hubbard model is introduced and equations for the GFs in the Nambu representation are derived. A self-consistent system of equations for GFs and the self-energy is formulated in Sec. III Results and discussion are presented in Sec. IV. Concluding remarks are given in Sec. V

\section{GENERAL FORMULATION}

\section{A. Extended Hubbard model}

We consider the extended Hubbard model on a square lattice

$$
\begin{aligned}
H & =\sum_{i \neq j, \sigma} t_{i j} a_{i \sigma}^{\dagger} a_{j \sigma}-\mu \sum_{i} N_{i} \\
& +(U / 2) \sum_{i} N_{i \sigma} N_{i \bar{\sigma}}+(1 / 2) \sum_{i \neq j} V_{i j} N_{i} N_{j}
\end{aligned}
$$

where $t_{i, j}$ is the single-electron hopping parameters, $a_{i \sigma}^{\dagger}$ and $a_{i \sigma}$ are the Fermi creation and annihilation operators for electrons with spin $\sigma / 2(\sigma= \pm 1=(\uparrow, \downarrow), \bar{\sigma}=-\sigma)$ on the lattice site $i, U$ is the on-site CI and the $V_{i j}$ is the intersite CI. $N_{i}=\sum_{\sigma} N_{i \sigma}, N_{i \sigma}=a_{i \sigma}^{\dagger} a_{i \sigma}$ is the number operator and $\mu$ is the chemical potential.

In the strong correlation limit the model describes the Mott-Hubbard insulating state at half-filling $\left(n=\left\langle N_{i}\right\rangle=\right.$ 1) when the conduction band splits into two Hubbard subbands. In this case the Fermi operators $a_{i \sigma}^{\dagger}, a_{i \sigma}$ in (1) fail to describe single-particle electron excitations in the system and the Fermi-liquid picture becomes inadequate for cuprates. The projected-type operators, the Hubbard operators (HOs), referring to the two subbands, singly occupied $a_{i \sigma}^{\dagger}\left(1-N_{i \bar{\sigma}}\right)$ and doubly occupied $a_{i \sigma}^{\dagger} N_{i \bar{\sigma}}$, must be introduced. In terms of the HOs the model (11) reads

$$
\begin{aligned}
H & =\varepsilon_{1} \sum_{i, \sigma} X_{i}^{\sigma \sigma}+\varepsilon_{2} \sum_{i} X_{i}^{22}+\frac{1}{2} \sum_{i \neq j} V_{i j} N_{i} N_{j} \\
& +\sum_{i \neq j, \sigma} t_{i j}\left\{X_{i}^{\sigma 0} X_{j}^{0 \sigma}+X_{i}^{2 \sigma} X_{j}^{\sigma 2}\right. \\
& \left.+\sigma\left(X_{i}^{2 \bar{\sigma}} X_{j}^{0 \sigma}+\text { H.c. }\right)\right\},
\end{aligned}
$$

where $\varepsilon_{1}=-\mu$ is the single-particle energy and $\varepsilon_{2}=$ $U-2 \mu$ is the two-particle energy. The matrix HOs $X_{i}^{\alpha \beta}=|i \alpha\rangle\langle i \beta|$ describes transition from the state $|i, \beta\rangle$ to the state $|i, \alpha\rangle$ on a lattice site $i$ taking into account four possible states for holes: an empty state $(\alpha, \beta=0)$, a singly occupied hole state $(\alpha, \beta=\sigma)$, and a doubly occupied hole state $(\alpha, \beta=2)$. The number operator and the spin operators in terms of the $\mathrm{HO}$ are defined as

$$
\begin{aligned}
N_{i} & =\sum_{\sigma} X_{i}^{\sigma \sigma}+2 X_{i}^{22} \\
S_{i}^{\sigma} & =X_{i}^{\sigma \bar{\sigma}}, \quad S_{i}^{z}=(\sigma / 2)\left[X_{i}^{\sigma \sigma}-X_{i}^{\bar{\sigma} \bar{\sigma}}\right] .
\end{aligned}
$$

The chemical potential $\mu$ is determined from the equation for an average occupation number for holes

$$
n=1+\delta=\left\langle N_{i}\right\rangle
$$

where $\langle\ldots\rangle$ denotes the statistical average with the Hamiltonian (2).

The HOs obey the completeness relation $X_{i}^{00}+$ $\sum_{\sigma} X_{i}^{\sigma \sigma}+X_{i}^{22}=1$ which rigorously preserves the constraint that only one quantum state $\alpha$ can be occupied on any lattice site $i$. The commutation relations for the $\mathrm{HOs}$

$$
\left[X_{i}^{\alpha \beta}, X_{j}^{\gamma \delta}\right]_{ \pm}=\delta_{i j}\left(\delta_{\beta \gamma} X_{i}^{\alpha \delta} \pm \delta_{\delta \alpha} X_{i}^{\gamma \beta}\right)
$$

with the upper sign for the Fermi-type operators (such as $\left.X_{i}^{0 \sigma}\right)$ and the lower sign for the Bose-type operators (such as $N_{i}$ (3) or the spin operators (4)) result in the so-called kinematic interaction. To demonstrate this let us consider the equation of motion for the $\mathrm{HO} X_{i}^{\sigma 2}=$ $a_{i \sigma}^{\dagger} a_{i \sigma} a_{i \bar{\sigma}}$ in the Heisenberg representation $(\hbar=1)$ :

$$
\begin{aligned}
i \frac{d}{d t} X_{i}^{\sigma 2} & =\left[X_{i}^{\sigma 2}, H\right]=\left(U-\mu+\sum_{l} V_{i l} N_{l}\right) X_{i}^{\sigma 2} \\
& +\sum_{l, \sigma^{\prime}} t_{i l}\left(B_{i \sigma \sigma^{\prime}}^{22} X_{l}^{\sigma^{\prime} 2}-\sigma B_{i \sigma \sigma^{\prime}}^{21} X_{l}^{0 \bar{\sigma}^{\prime}}\right) \\
& -\sum_{l} t_{i l} X_{i}^{02}\left(X_{l}^{\sigma 0}+\sigma X_{l}^{2 \bar{\sigma}}\right),
\end{aligned}
$$

Here $B_{i \sigma \sigma^{\prime}}^{\eta \zeta}$ are the Bose-type operators,

$$
\begin{aligned}
B_{i \sigma \sigma^{\prime}}^{22} & =\left(X_{i}^{22}+X_{i}^{\sigma \sigma}\right) \delta_{\sigma^{\prime} \sigma}+X_{i}^{\sigma \bar{\sigma}} \delta_{\sigma^{\prime} \bar{\sigma}} \\
& =\left(N_{i} / 2+\sigma S_{i}^{z}\right) \delta_{\sigma^{\prime} \sigma}+S_{i}^{\sigma} \delta_{\sigma^{\prime} \bar{\sigma}}, \\
B_{i \sigma \sigma^{\prime}}^{21} & =\left(N_{i} / 2+\sigma S_{i}^{z}\right) \delta_{\sigma^{\prime} \sigma}-S_{i}^{\sigma} \delta_{\sigma^{\prime} \bar{\sigma}} .
\end{aligned}
$$

We see that the hopping amplitudes depend on the number operator (3) and the spin operators (4) which results in the kinematic interaction describing effective scattering of electrons on spin and charge fluctuations. In phenomenological models for cuprates a dynamical coupling of electrons with spin and charge fluctuations is introduced specified by fitting parameters, while in Eq. (77) the interaction is determined by the hopping energy $t_{i j}$ fixed by the electronic dispersion. 


\section{B. Green functions}

To consider superconducting pairing in the model (2), we introduce the two-time thermodynamic GF [21] expressed in terms of the four-component Nambu operators, $\hat{X}_{i \sigma}$ and $\hat{X}_{i \sigma}^{\dagger}=\left(\begin{array}{llll}X_{i}^{2 \sigma} & X_{i}^{\bar{\sigma} 0} X_{i}^{\bar{\sigma} 2} X_{i}^{0 \sigma}\end{array}\right)$ :

$$
\begin{aligned}
\mathrm{G}_{i j \sigma}\left(t-t^{\prime}\right) & =-i \theta\left(t-t^{\prime}\right)\left\langle\left\{\hat{X}_{i \sigma}(t), \hat{X}_{j \sigma}^{\dagger}\left(t^{\prime}\right)\right\}\right\rangle \\
& \equiv\left\langle\left\langle\hat{X}_{i \sigma}(t) \mid \hat{X}_{j \sigma}^{\dagger}\left(t^{\prime}\right)\right\rangle\right\rangle,
\end{aligned}
$$

where $\{A, B\}=A B+B A, A(t)=\exp (i H t) A \exp (-i H t)$, and $\theta(x)=1$ for $x>0$ and $\theta(x)=0$ for $x<0$. The Fourier representation in $(\mathbf{k}, \omega)$-space is defined by the relations:

$$
\begin{aligned}
\mathrm{G}_{i j \sigma}\left(t-t^{\prime}\right) & =\frac{1}{2 \pi} \int_{-\infty}^{\infty} d t e^{-i\left(t-t^{\prime}\right)} \mathrm{G}_{i j \sigma}(\omega), \\
\mathrm{G}_{i j \sigma}(\omega) & =\frac{1}{N} \sum_{\mathbf{k}} \exp [i \mathbf{k}(\mathbf{i}-\mathbf{j})] \mathrm{G}_{\sigma}(\mathbf{k}, \omega) .
\end{aligned}
$$

The GF (12) is convenient to write in the matrix form

$$
\mathrm{G}_{\sigma}(\mathbf{k}, \omega)=\left(\begin{array}{cc}
\hat{G}_{\sigma}(\mathbf{k}, \omega) & \hat{F}_{\sigma}(\mathbf{k}, \omega) \\
\hat{F}_{\sigma}^{\dagger}(\mathbf{k}, \omega) & -\hat{G}_{\bar{\sigma}}(-\mathbf{k},-\omega)
\end{array}\right)
$$

where the normal $\hat{G}_{\sigma}(\mathbf{k}, \omega)$ and anomalous (pair) $\hat{F}_{\sigma}(\mathbf{k}, \omega)$ GFs are $2 \times 2$ matrices for two Hubbard subbands:

$$
\begin{aligned}
& \hat{G}_{\sigma}(\mathbf{k}, \omega)=\left\langle\left\langle\left(\begin{array}{c}
X_{\mathbf{k}}^{\sigma 2} \\
X_{\mathbf{k}}^{0 \bar{\sigma}}
\end{array}\right) \mid X_{\mathbf{k}}^{2 \sigma} X_{\mathbf{k}}^{\bar{\sigma} 0}\right\rangle_{\omega},\right. \\
& \hat{F}_{\sigma}(\mathbf{k}, \omega)=\left\langle\left\langle\left(\begin{array}{c}
X_{\mathbf{k}}^{\sigma 2} \\
X_{\mathbf{k}}^{0 \bar{\sigma}}
\end{array}\right) \mid X_{-\mathbf{k}}^{\bar{\sigma} 2} X_{-\mathbf{k}}^{0 \sigma}\right\rangle_{\omega} .\right.
\end{aligned}
$$

To calculate the GF (10) we use the equation of motion method by differentiating the GF with respect to time $t$ and $t^{\prime}$. As described in detail in Refs. [18, 22], using the Mori-type projection method [20] we derive an exact representation for the GF (13) in the form of the Dyson equation

$$
\mathrm{G}_{\sigma}(\mathbf{k}, \omega)=\left[\omega \tilde{\tau}_{0}-\mathrm{E}_{\sigma}(\mathbf{k})-\mathrm{Q} \Sigma_{\sigma}(\mathbf{k}, \omega)\right]^{-1} \mathrm{Q},
$$

where $\tilde{\tau}_{0}$ is the $4 \times 4$ unit matrix. The electron excitation spectrum in the generalized mean-field approximation (GMFA) is determined by the time-independent matrix of correlation functions:

$$
\begin{aligned}
\mathrm{E}_{\sigma}(\mathbf{k}) & =\frac{1}{N} \sum_{\mathbf{k}} \exp [i \mathbf{k}(\mathbf{i}-\mathbf{j})]\left\langle\left\{\left[\hat{X}_{i \sigma}, H\right], \hat{X}_{j \sigma}^{\dagger}\right\}\right\rangle \mathrm{Q}^{-1} \\
& =\left(\begin{array}{cc}
\hat{\varepsilon}(\mathbf{k}) & \hat{\Delta}_{\sigma}(\mathbf{k}) \\
\hat{\Delta}_{\sigma}^{*}(\mathbf{k}) & -\hat{\varepsilon}_{\bar{\sigma}}(\mathbf{k})
\end{array}\right)
\end{aligned}
$$

where $\hat{\varepsilon}(\mathbf{k})$ and $\hat{\Delta}_{\sigma}(\mathbf{k})$ are the normal and anomalous parts of the energy matrix. The parameter $\mathrm{Q}=$
$\left\langle\left\{\hat{X}_{i \sigma}, \hat{X}_{i \sigma}^{\dagger}\right\}\right\rangle=\hat{\tau}_{0} \times \hat{Q}$ where $\hat{\tau}_{0}$ is the $2 \times 2$ unit matrix and $\hat{Q}=\left(\begin{array}{cc}Q_{2} & 0 \\ 0 & Q_{1}\end{array}\right)$ takes into account a redistribution of the spectral weights with doping of the Hubbard subbands $Q_{2}=\left\langle X_{i}^{22}+X_{i}^{\sigma \sigma}\right\rangle=n / 2$ and $Q_{1}=\left\langle X_{i}^{00}+X_{i}^{\bar{\sigma} \bar{\sigma}}\right\rangle=1-Q_{2}$.

The self-energy operator in Eq. (16)

$$
\mathrm{Q} \Sigma_{\sigma}(\mathbf{k}, \omega)=\left\langle\left\langle\hat{Z}_{\mathbf{k} \sigma}^{(\mathrm{ir})} \mid \hat{Z}_{\mathbf{k} \sigma}^{(\mathrm{ir}) \dagger}\right\rangle\right\rangle_{\omega}^{(\mathrm{pp})} \mathrm{Q}^{-1},
$$

determined by irreducible operators $\hat{Z}_{i \sigma}^{(\mathrm{ir})}=\left[\hat{X}_{i \sigma}, H\right]-$ $\sum_{l} \mathrm{E}_{i l \sigma} \hat{X}_{l \sigma}$, describes processes of inelastic scattering of electrons (holes) on spin and charge fluctuations due to the kinematic interaction and CI $V_{i j}$ (see Eq. (17)). The self-energy operator (18) can be written in the same matrix form as the GF (13):

$$
\mathrm{Q} \Sigma_{\sigma}(\mathbf{k}, \omega)=\left(\begin{array}{cc}
\hat{M}_{\sigma}(\mathbf{k}, \omega) & \hat{\Phi}_{\sigma}(\mathbf{k}, \omega) \\
\hat{\Phi}_{\sigma}^{\dagger}(\mathbf{k}, \omega) & -\hat{M}_{\bar{\sigma}}(\mathbf{k},-\omega)
\end{array}\right) \mathrm{Q}^{-1}
$$

where the matrices $\hat{M}$ and $\hat{\Phi}$ denote the respective normal and anomalous (pair) components of the self-energy operator.

The system of equations for the $(4 \times 4)$ matrix GF (13) and the self-energy (19) can be reduced to a system of equations for the normal $\hat{G}_{\sigma}(\mathbf{k}, \omega)$ and the pair $\hat{F}_{\sigma}(\mathbf{k}, \omega)$ $(2 \times 2)$ matrix components. Using representations for the energy matrix (17) and the self-energy (19), we derive for these components the following system of matrix equations:

$$
\begin{aligned}
\hat{G}(\mathbf{k}, \omega) & =\left(\hat{G}_{N}(\mathbf{k}, \omega)^{-1}\right. \\
& \left.+\hat{\varphi}_{\sigma}(\mathbf{k}, \omega) \hat{G}_{N}(\mathbf{k},-\omega) \hat{\varphi}_{\sigma}^{*}(\mathbf{k}, \omega)\right)^{-1} \hat{Q}, \\
\hat{F}_{\sigma}(\mathbf{k}, \omega) & =-\hat{G}_{N}(\mathbf{k},-\omega) \hat{\varphi}_{\sigma}(\mathbf{k}, \omega) \hat{G}(\mathbf{k}, \omega),
\end{aligned}
$$

where we introduced the normal state GF

$$
\hat{G}_{N}(\mathbf{k}, \omega)=\left(\omega \hat{\tau}_{0}-\hat{\varepsilon}(\mathbf{k})-\hat{M}(\mathbf{k}, \omega) / \hat{Q}\right)^{-1},
$$

and the superconducting gap function

$$
\hat{\varphi}_{\sigma}(\mathbf{k}, \omega)=\hat{\Delta}_{\sigma}(\mathbf{k})+\hat{\Phi}_{\sigma}(\mathbf{k}, \omega) / \hat{Q}
$$

The Dyson equation (16) with the zero-order quasiparticle (QP) excitation energy (17) and the self-energy (19) gives an exact representation for the GF (10). To obtain a closed system of equations, the multiparticle GF in the self-energy operator (18) should be evaluated as discussed below.

\section{APPROXIMATE SYSTEM OF EQUATIONS}

In this section we derive an approximate system of equations for the GFs and the self-energy components in Eqs. (20) - (23) for the two Hubbard subbands adopting several approximations to make the system of equations numerically tractable. 


\section{A. Generalized mean-field approximation}

The energy matrix (17) is calculated using the commutation relations (6) for the HOs. The normal part of the energy matrix $\hat{\varepsilon}(\mathbf{k})$ after diagonalization determines the QP spectrum in two Hubbard subbands in the GMFA (for detail see 22]):

$$
\begin{aligned}
\varepsilon_{1,2}(\mathbf{k}) & =(1 / 2)\left[\omega_{2}(\mathbf{k})+\omega_{1}(\mathbf{k})\right] \mp(1 / 2) \Lambda(\mathbf{k}) \\
\omega_{\iota}(\mathbf{k}) & =4 t \alpha_{\iota} \gamma(\mathbf{k})+4 \beta_{\iota} t^{\prime} \gamma^{\prime}(\mathbf{k})+4 \beta_{\iota} t^{\prime \prime} \gamma^{\prime \prime}(\mathbf{k}) \\
& +\omega_{\iota}^{(c)}(\mathbf{k})+U \delta_{\iota, 2}-\mu, \quad(\iota=1,2) \\
\Lambda(\mathbf{k}) & =\left\{\left[\omega_{2}(\mathbf{k})-\omega_{1}(\mathbf{k})\right]^{2}+4 W(\mathbf{k})^{2}\right\}^{1 / 2} \\
W(\mathbf{k}) & =4 t \alpha_{12} \gamma(\mathbf{k})+4 t^{\prime} \beta_{12} \gamma^{\prime}(\mathbf{k})+4 t^{\prime \prime} \beta_{12} \gamma^{\prime \prime}(\mathbf{k})
\end{aligned}
$$

Here the hopping parameter is defined by the expression:

$$
\begin{aligned}
t_{i j} & =(1 / N) \sum_{\mathbf{k}} \exp [i \mathbf{k}(\mathbf{i}-\mathbf{j})] t(\mathbf{k}), \\
t(\mathbf{k}) & =4 t \gamma(\mathbf{k})+4 t^{\prime} \gamma^{\prime}(\mathbf{k})+4 t^{\prime \prime} \gamma^{\prime \prime}(\mathbf{k}),
\end{aligned}
$$

where the nearest-neighbor hopping is $t$, diagonal hopping is $t^{\prime}$ and the third neighbor hopping is $t^{\prime \prime}$. The corresponding $\mathbf{k}$-dependent functions are: $\gamma(\mathbf{k})=$ $(1 / 2)\left(\cos k_{x}+\cos k_{y}\right), \quad \gamma^{\prime}(\mathbf{k})=\cos k_{x} \cos k_{y}$, and $\gamma^{\prime \prime}(\mathbf{k})=(1 / 2)\left(\cos 2 k_{x}+\cos 2 k_{y}\right)$ (the lattice constants $a_{x}=a_{y}$ are put to unity). The contribution from the CI $V_{i j}$ in (25) is given by

$$
\omega_{1(2)}^{(c)}(\mathbf{k})=\frac{1}{N} \sum_{\mathbf{q}} V(\mathbf{k}-\mathbf{q}) N_{1(2)}(\mathbf{q}),
$$

where $N_{1}(\mathbf{q})=\left\langle X_{\mathbf{q}}^{0 \bar{\sigma}} X_{\mathbf{q}}^{\bar{\sigma} 0}\right\rangle / Q_{1}$ and $N_{2}(\mathbf{q})=$ $\left\langle X_{\mathbf{q}}^{\sigma 2} X_{\mathbf{q}}^{2 \sigma}\right\rangle / Q_{2}$ are occupation numbers in the singleparticle and two-particle subbands, respectively. $V(\mathbf{q})$ is the Fourier transform of $V_{i j}$.

The kinematic interaction for the HOs results in renormalization of the spectrum (24) determined by the parameters: $\alpha_{\iota}=Q_{\iota}\left[1+C_{1} / Q_{\iota}^{2}\right], \beta_{\iota}=Q_{\iota}[1+$ $\left.C_{2} / Q_{\iota}^{2}\right], \alpha_{12}=\sqrt{Q_{1} Q_{2}}\left[1-C_{1} / Q_{1} Q_{2}\right], \beta_{12}=\sqrt{Q_{1} Q_{2}}[1-$ $\left.C_{2} / Q_{1} Q_{2}\right]$. In addition to the conventional Hubbard I renormalization given by $Q_{1}, Q_{2}$ parameters an essential renormalization is caused by the AF spin correlation functions for nearest-neighbors and next neighbors, respectively:

$$
C_{1}=\left\langle\mathbf{S}_{i} \mathbf{S}_{i+a_{1}}\right\rangle, \quad C_{2}=\left\langle\mathbf{S}_{i} \mathbf{S}_{i+a_{2}}\right\rangle
$$

These functions strongly depend on doping resulting in a considerable variation of the electronic spectrum as shown later and discussed in detail in Ref. [22].

The anomalous component $\hat{\Delta}_{\sigma}(\mathbf{k})$ of the matrix (17) determines the superconduction gap in the GMFA. The diagonal matrix components in the coordinate representation are given by the equations:

$$
\begin{aligned}
& \Delta_{i j \sigma}^{22} Q_{2}=-\sigma t_{i j}^{21}\left\langle X_{i}^{02} N_{j}\right\rangle-V_{i j}\left\langle X_{i}^{\sigma 2} X_{j}^{\bar{\sigma} 2}\right\rangle \\
& \Delta_{i j \sigma}^{11} Q_{1}=\sigma t_{i j}^{12}\left\langle N_{j} X_{i}^{02}\right\rangle-V_{i j}\left\langle X_{i}^{0 \bar{\sigma}} X_{j}^{0 \sigma}\right\rangle
\end{aligned}
$$

Here we introduced upper indexes for the hopping parameter $t_{i j}^{12}, t_{i j}^{21}$ to stress that the anomalous components $\left\langle X_{i}^{02} N_{j}\right\rangle$ are induced by the interband hopping. Calculation of the correlation function $\left\langle X_{i}^{02} N_{j}\right\rangle$ from the equation of motion for the GF $L_{i j}\left(t-t^{\prime}\right)=\left\langle\left\langle X_{i}^{02}(t) \mid N_{j}\left(t^{\prime}\right)\right\rangle\right\rangle$ results in the superconducting gap in the two-particle subband (for detail see Ref. [23]):

$$
\Delta_{i j \sigma}^{22}=\left(J_{i j}-V_{i j}\right)\left\langle X_{i}^{\sigma 2} X_{j}^{\bar{\sigma} 2}\right\rangle / Q_{2},
$$

where $J_{i j}=4\left(t_{i j}^{12}\right)^{2} / U$ is the AF exchange interaction. A similar equation holds for the gap in the single-particle subband: $\Delta_{i j \sigma}^{11}=\left(J_{i j}-V_{i j}\right)\left\langle X_{i}^{0 \bar{\sigma}} X_{j}^{0 \sigma}\right\rangle / Q_{1}$. Therefore, the pairing in the Hubbard model in the GMFA is similar to the superconductivity in the $t-J$ model mediated by the AF exchange interaction $J_{i j}$.

\section{B. Self-energy operator}

The self-energy matrix (19) due to the kinematic interaction, as shown in Eq. (7), is determined by multiparticle GFs such as $\left\langle\left\langle\hat{X}_{l \sigma^{\prime}}(t) B_{i \sigma \sigma^{\prime}}(t) \mid \hat{X}_{l^{\prime} \sigma^{\prime \prime}}^{\dagger} B_{j \sigma \sigma^{\prime \prime}}^{\dagger}\right\rangle\right.$. We calculate the self-energy matrix in the SCBA using the mode-coupling approximation for the multiparticle GFs. In this approximation, a propagation of excitations described by the Fermi-like operators $\hat{X}_{l \sigma}$ and the Bose-like operators $B_{i \sigma \sigma^{\prime}}$ for $l \neq i$ is assumed to be independent. Therefore, the corresponding time-dependent multiparticle correlation functions can be written as a product of fermionic and bosonic correlation functions,

$$
\begin{aligned}
& \left\langle X_{l^{\prime}}^{2 \sigma^{\prime \prime}} B_{j \sigma \sigma^{\prime \prime}}^{\dagger} \mid B_{i \sigma \sigma^{\prime}}(t) X_{l}^{\sigma^{\prime} 2}(t)\right\rangle \\
& =\delta_{\sigma^{\prime}, \sigma^{\prime \prime}}\left\langle X_{l^{\prime}}^{2 \sigma^{\prime}} X_{l}^{\sigma^{\prime} 2}(t)\right\rangle\left\langle B_{j \sigma \sigma^{\prime}}^{\dagger} \mid B_{i \sigma \sigma^{\prime}}(t)\right\rangle, \\
& \left\langle X_{l^{\prime}}^{\bar{\sigma}^{\prime \prime} 2} B_{j \bar{\sigma} \bar{\sigma}^{\prime \prime}} \mid B_{i \sigma \sigma^{\prime}}(t) X_{l}^{\sigma^{\prime} 2}(t)\right\rangle \\
& =\delta_{\sigma^{\prime}, \sigma^{\prime \prime}}\left\langle X_{l^{\prime}}^{\bar{\sigma}^{\prime} 2} X_{l}^{\sigma^{\prime} 2}(t)\right\rangle\left\langle B_{j \bar{\sigma} \bar{\sigma}^{\prime}} B_{i \sigma \sigma^{\prime}}(t)\right\rangle .
\end{aligned}
$$

The time-dependent single-particle correlation functions are calculated self-consistently using the corresponding GFs. This approximation results in a self-consistent system of equations for the self-energy (19) and the GFs (20), (21) similar to the strong-coupling Eliashberg theory [24] (for detail see Ref. [18] and Chapter A in Ref. [2]).

In this approximation the normal state GF (22) for two subbands takes the form [22]:

$$
\begin{aligned}
G_{N}^{11(22)}(\mathbf{k}, \omega) & =[1-b(\mathbf{k})] G_{1(2)}(\mathbf{k}, \omega) \\
& +b(\mathbf{k}) G_{2(1)}(\mathbf{k}, \omega) \\
G_{1(2)}(\mathbf{k}, \omega) & =\frac{1}{\omega-\varepsilon_{1(2)}(\mathbf{k})-\Sigma(\mathbf{k}, \omega)},
\end{aligned}
$$

where the hybridization parameter $b(\mathbf{k})=\left[\varepsilon_{2}(\mathbf{k})-\right.$ $\left.\omega_{2}(\mathbf{k})\right] /\left[\varepsilon_{2}(\mathbf{k})-\varepsilon_{1}(\mathbf{k})\right]$. The self-energy $\Sigma(\mathbf{k}, \omega)$ can be approximated by the same function for two subbands. In the imaginary frequency representation, $i \omega_{n}=i \pi T(2 n+$ 
1), $n=0, \pm 1, \pm 2, \ldots$ it reads

$$
\begin{aligned}
\Sigma\left(\mathbf{k}, \omega_{n}\right) & =-\frac{T}{N} \sum_{\mathbf{q}} \sum_{m} \lambda^{(+)}\left(\mathbf{q}, \mathbf{k}-\mathbf{q} \mid \omega_{n}-\omega_{m}\right) \\
& \times\left[G_{1}\left(\mathbf{q}, \omega_{m}\right)+G_{2}\left(\mathbf{q}, \omega_{m}\right)\right] \\
& \equiv i \omega_{n}\left[1-Z\left(\mathbf{k}, \omega_{n}\right)\right]+X\left(\mathbf{k}, \omega_{n}\right) .
\end{aligned}
$$

The normal GF (36) for the two subbands takes the form:

$$
\begin{array}{r}
\left\{G_{1(2)}\left(\mathbf{k}, \omega_{n}\right)\right\}^{-1}=i \omega_{n}-\varepsilon_{1(2)}(\mathbf{k})-\Sigma\left(\mathbf{k}, \omega_{n}\right) \\
=i \omega_{n} Z\left(\mathbf{k}, \omega_{n}\right)-\left[\varepsilon_{1(2)}(\mathbf{k})+X\left(\mathbf{k}, \omega_{n}\right)\right] .
\end{array}
$$

To calculate $T_{c}$ we can use a linear approximation for the pair GF (21). In particular, Eq. (23) for the two-particle subband gap $\varphi(\mathbf{k}, \omega)=\sigma \varphi_{2, \sigma}(\mathbf{k}, \omega)$ can be written as

$$
\begin{aligned}
\varphi\left(\mathbf{k}, \omega_{n}\right) & =\frac{T_{c}}{N} \sum_{\mathbf{q}} \sum_{m}\{J(\mathbf{k}-\mathbf{q})-V(\mathbf{k}-\mathbf{q}) \\
& \left.+\lambda^{(-)}\left(\mathbf{q}, \mathbf{k}-\mathbf{q} \mid \omega_{n}-\omega_{m}\right)\right\} \\
& \times \frac{[1-b(\mathbf{q})]^{2} \varphi\left(\mathbf{q}, \omega_{m}\right)}{\left.\left[\omega_{m} Z\left(\mathbf{q}, \omega_{m}\right)\right]^{2}+\left[\varepsilon_{2}(\mathbf{q})+X \mathbf{q}, \omega_{m}\right)\right]^{2}} .
\end{aligned}
$$

The interaction functions in (37) and (39) in the imaginary frequency representation are given by

$$
\begin{aligned}
& \lambda^{( \pm)}\left(\mathbf{q}, \mathbf{k}-\mathbf{q} \mid \nu_{n}\right)=-|t(\mathbf{q})|^{2} \chi_{s f}\left(\mathbf{k}-\mathbf{q}, \nu_{n}\right) \\
& \mp\left\{|V(\mathbf{k}-\mathbf{q})|^{2}+|t(\mathbf{q})|^{2} / 4\right\} \chi_{c f}\left(\mathbf{k}-\mathbf{q}, \nu_{n}\right) .
\end{aligned}
$$

The spectral densities of bosonic excitations are determined by the dynamic susceptibility for spin $(s f)$ and number (charge) ( $c f$ ) fluctuations

$$
\begin{aligned}
& \chi_{s f}(\mathbf{q}, \omega)=-\left\langle\left\langle\mathbf{S}_{\mathbf{q}} \mid \mathbf{S}_{-\mathbf{q}}\right\rangle\right\rangle_{\omega}, \\
& \chi_{c f}(\mathbf{q}, \omega)=-\left\langle\left\langle\delta N_{\mathbf{q}} \mid \delta N_{-\mathbf{q}}\right\rangle\right\rangle_{\omega},
\end{aligned}
$$

written in terms of the commutator GFs [21] for spin $\mathbf{S}_{\mathbf{q}}$ and number $\delta N_{\mathbf{q}}=N_{\mathbf{q}}-\left\langle N_{\mathbf{q}}\right\rangle$ operators.

Thus, we have derived the self-consistent system of equations for the normal GF (38), the self-energy (37), and the gap function (39). In the SCBA, vertex corrections to the kinematic interaction $t(\mathbf{q})$ of electrons with spin- and charge-fluctuations (411), (42) induced by the intraband hopping are neglected. It is assumed that the system is far away from a charge instability or a stripe formation and charge-fluctuations give a small contribution to the pairing. The largest contribution from spin fluctuations comes from wave-vectors close the $\mathrm{AF}$ wave-vector $\mathbf{Q}=(\pi, \pi)$ where their energy $\omega_{s}(\mathbf{Q})$ is much smaller than the Fermi energy, $\omega_{s}(\mathbf{Q}) / \mu \ll 1$ (see, e.g., 26]). Therefore, vertex corrections to the kinematic interaction should be small as in Eliashberg theory 24] for electron interaction with phonons, where $\omega_{p h}(\mathbf{q}) / \mu \ll 1$. Consequently, the SCBA for the selfenergy and the GFs calculated self-consistently is quite reliable and makes it possible to consider the strong coupling regime which is essential in study of renormalization of the QP spectrum and the superconducting pairing as shown in Refs. [18, 22] and discussed later.

\section{RESULTS AND DISCUSSION}

In numerical computations we have used models for the CIs and the susceptibility (41), (42). For the intersite CI $V_{i j}$ we consider a model for repulsion of two electrons (holes) on neighbor lattice sites,

$$
V(\mathbf{q})=2 V\left(\cos q_{x}+\cos q_{y}\right),
$$

with various values of $V=0.0,0.5 t, 1.0 t$ and $2.0 t$. For the on-site CI we consider $U=8 t, 16 t$ and $32 t$. The AF exchange interaction for neighbor sites is described by the function $J(\mathbf{q})=2 J\left(\cos q_{x}+\cos q_{y}\right)$. Note, that in the GMFA the CI $V_{i j}$ gives no contribution to the exchange interaction $J_{i j}$ and therefore it is assumed to be the same for all values of $V$ (cf. with Refs. [15, 16]). In the most of calculations we take $J=0.4 t$ but to study a role of the spin-fluctuation interaction in the superconducting pairing, we consider also other values of the interaction, $J=0.2 t, 0.6 t$, and $1.0 t$.

Due to a large energy scale of charge fluctuations, of the order of several $t$, in comparison with the spin excitation energy of the order of $J$, the charge fluctuation contributions can be considered in the static limit for the susceptibility (42)

$$
\begin{aligned}
\chi_{c f}(\mathbf{k}) & =\chi_{c f}^{(1)}(\mathbf{k})+\chi_{c f}^{(2)}(\mathbf{k}), \\
\chi_{c f}^{(\alpha)}(\mathbf{k}) & =-\frac{1}{N} \sum_{\mathbf{q}} \frac{N^{(\alpha)}(\mathbf{q}+\mathbf{k})-N^{(\alpha)}(\mathbf{q})}{\varepsilon_{\alpha}(\mathbf{q}+\mathbf{k})-\varepsilon_{\alpha}(\mathbf{q})},
\end{aligned}
$$

where the occupation numbers $N^{(\alpha)}(\mathbf{q})$ are defined as

$$
\begin{aligned}
N^{(1)}(\mathbf{k}) & =\left[Q_{1}+(n-1) b(\mathbf{k})\right] N_{1}(\mathbf{k}), \\
N^{(2)}(\mathbf{k}) & =\left[Q_{2}-(n-1) b(\mathbf{k})\right] N_{2}(\mathbf{k}), \\
N_{\alpha}(\mathbf{k}) & =(1 / 2)+T \sum_{m} G_{\alpha}\left(\mathbf{k}, \omega_{m}\right) .
\end{aligned}
$$

For the dynamical spin susceptibility $\chi_{s f}(\mathbf{q}, \omega)$ (41) we used a model suggested in Ref. [25]

$$
\begin{gathered}
\operatorname{Im} \chi_{s f}\left(\mathbf{q}, \omega+i 0^{+}\right)=\chi_{s f}(\mathbf{q}) \chi_{s f}^{\prime \prime}(\omega) \\
=\frac{\chi_{Q}}{1+\xi^{2}[1+\gamma(\mathbf{q})]} \tanh \frac{\omega}{2 T} \frac{1}{1+\left(\omega / \omega_{s}\right)^{2}} .
\end{gathered}
$$

This type of the spin-excitation spectrum was found in the microscopic theory for the $t-J$ model in Ref. [26]. The model is determined by two parameters: the $\mathrm{AF}$ correlation length $\xi$ and the cut-off energy of spin excitations of the order of the exchange energy $\omega_{s} \sim J$. The strength of the spin-fluctuation interaction given by the static susceptibility $\chi_{Q}=\chi_{s f}(\mathbf{Q})$ at the $\mathrm{AF}$ wave vector $\mathbf{Q}=(\pi, \pi)$,

$$
\chi_{Q}=\frac{3(1-\delta)}{2 \omega_{s}}\left\{\frac{1}{N} \sum_{\mathbf{q}} \frac{1}{1+\xi^{2}[1+\gamma(\mathbf{q})]}\right\}^{-1},
$$


is defined by the normalization condition:

$$
\frac{1}{N} \sum_{\mathbf{q}} \int_{0}^{\infty} \frac{d \omega}{\pi} \operatorname{coth} \frac{\omega}{2 T} \operatorname{Im} \chi_{s f}(\mathbf{q}, \omega)=\left\langle\mathbf{S}_{i}^{2}\right\rangle=\frac{3}{4}(1-\delta) .
$$

The spin correlation functions (29) in the singleparticle excitation spectrum (24) are calculated using the same model (46): $C_{1}=(1 / N) \sum_{\mathbf{q}} C_{\mathbf{q}} \gamma(\mathbf{q}), C_{2}=$ $(1 / N) \sum_{\mathbf{q}} C_{\mathbf{q}} \gamma^{\prime}(\mathbf{q})$, where $C_{\mathbf{q}}=\left(\omega_{s} / 2\right)\left(\chi_{Q} /\left\{1+\xi^{2}[1+\right.\right.$ $\gamma(\mathbf{q})]\})$. As an energy unit we use $t=0.4 \mathrm{eV}$ and for the hopping parameters we take $t^{\prime}=-0.2 t, \quad t^{\prime \prime}=0.10 t$. Below we present numerical results for a hole-doped case for the two-hole subband.

\section{A. Electronic spectrum in the normal state}

At first we consider results in the GMFA for the electronic spectrum (24). The doping dependence of the electron dispersion for the two-hole subband $\varepsilon_{2}(\mathbf{k})$ along the symmetry directions in the $2 \mathrm{D}$ Brillouin zone (BZ) are shown in Fig. 1 for $U=8$ and in Fig. 2 for $U=16$ for $V=0$ (a) and for $V=2$ (b). The corresponding Fermi surfaces (FSs) determined by the equation: $\varepsilon_{2}\left(\mathbf{k}_{\mathrm{F}}\right)=0$ are plotted in Fig. 3 and Fig. [4. For small doping, $\delta=0.05$, the energy at the $M(\pi, \pi)$ and $\Gamma(0,0)$ points are nearly equal as in the AF phase. Only small hole-like FS pockets close to the $( \pm \pi / 2, \pm \pi / 2)$ points emerge at this doping as shown in Figs. 3, 4. With increasing doping, the AF correlation length decreases that results in increasing of the electron energy at the $M(\pi, \pi)$ point and at some critical doping $\delta \sim 0.12$ a large FS appears. At the same time, the renormalized two-hole subband width increases with doping, as e.g. for $U=8$ and $V=0$ from $\widetilde{W} \approx 2 t$ at $\delta=0.05$ to $\widetilde{W} \approx 3 t$ at $\delta=0.25$, which, however, remains less than the "bare" Hubbard subband width $W=4 t(1+\delta)$ where short-range AF correlations are disregarded. With increasing CI $U$ and $V$ the subband width shrinks as seen from comparison panels (a) and (b) for electronic spectra in Figs. 1, 2 and the FS in Figs. 3, 4.

To study self-energy effects in the electronic spectrum the strong coupling theory (SCT) should be used as a self-consistent solution of the system of equations for the normal GF (36) and the self-energy (37). Since detailed investigation of the normal state electronic spectrum in SCT was performed for the conventional Hubbard model in Ref. [22] and for the extended Hubbard model in Ref. [18], here we present results only for $U$ and $V$ dependence of the renormalization parameter $Z(\mathbf{q})$ at the Fermi energy

$$
\begin{aligned}
Z(\mathbf{q}) & =Z(\mathbf{q}, \omega=0)=1+\lambda(\mathbf{q}) \\
& =1-\left.[d \operatorname{Re} \Sigma(\mathbf{q}, \omega) / d \omega]\right|_{\omega=0} .
\end{aligned}
$$

We found that $Z(\mathbf{q})$ weakly depends on $\delta$ for $\delta \lesssim 0.15$ (see also Ref. [18]). Therefore, in Fig. [5 we demonstrate
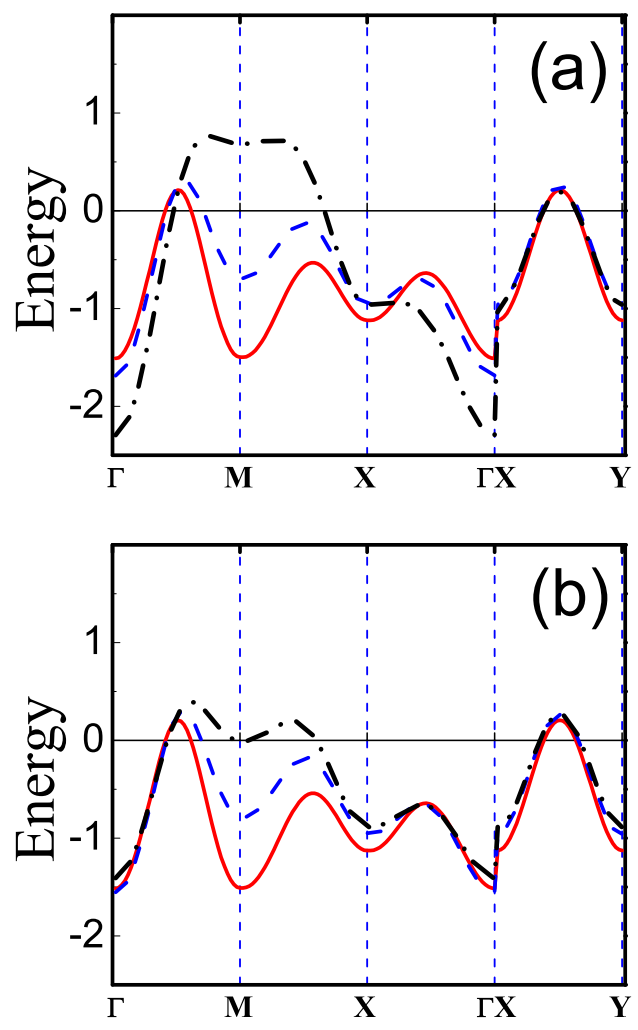

FIG. 1: (Color online) Electron dispersion in the GMFA $\varepsilon_{2}(\mathbf{k})$ for (a) $V=0$ and (b) $V=2$ at $U=8$ along the symmetry directions $\Gamma(0,0) \rightarrow M(\pi, \pi) \rightarrow X(\pi, 0) \rightarrow \Gamma(0,0)$ and $X(\pi, 0) \rightarrow Y(0, \pi)$ for $\delta=0.05$ (red solid line), 0.10 (blue dashed line), and 0.25 (black dash-dotted line). Fermi energy for hole doping is at $\omega=0$.

the $U$ dependence of $Z(\mathbf{q})$ at $\delta=0.10$ for $V=0$ (a) and for $V=1$ (b). It appears that the renormalization parameter $Z(\mathbf{q})$ is quite large in the whole $\mathrm{BZ}, Z(\mathbf{q}) \sim$ $4-6$, which results in a strong suppression of the QP weight $\sim 1 / Z(\mathbf{q})$.

\section{B. Superconducting $\mathbf{T}_{\mathrm{c}}$}

For a comparison of various contributions to the superconducting gap equation (39), we approximate the interaction (40) by its value close to the Fermi energy. As the result instead of the dynamical susceptibility (41), (42) the static susceptibility $\chi(\mathbf{q})=\operatorname{Re} \chi(\mathbf{q}, \Omega=0)$ appears in the gap equation. It brings us to the BCS-type equation for the gap function (39) at the Fermi energy $\varphi(\mathbf{k})=\varphi(\mathbf{k}, \omega=0)$ :

$$
\begin{aligned}
& \varphi(\mathbf{k})=\frac{1}{N} \sum_{\mathbf{q}} \frac{[1-b(\mathbf{q})]^{2} \varphi(\mathbf{q})}{[Z(\mathbf{q})]^{2} 2 \widetilde{\varepsilon}(\mathbf{q})} \tanh \frac{\widetilde{\varepsilon}(\mathbf{q})}{2 T_{c}}\{J(\mathbf{k}-\mathbf{q}) \\
& -V(\mathbf{k}-\mathbf{q})+\left[(1 / 4)|t(\mathbf{q})|^{2}+|V(\mathbf{k}-\mathbf{q})|^{2}\right] \chi_{c f}(\mathbf{k}-\mathbf{q}) \\
& \left.-|t(\mathbf{q})|^{2} \chi_{s f}(\mathbf{k}-\mathbf{q}) \theta\left(\omega_{s}-|\widetilde{\varepsilon}(\mathbf{q})|\right)\right\},
\end{aligned}
$$



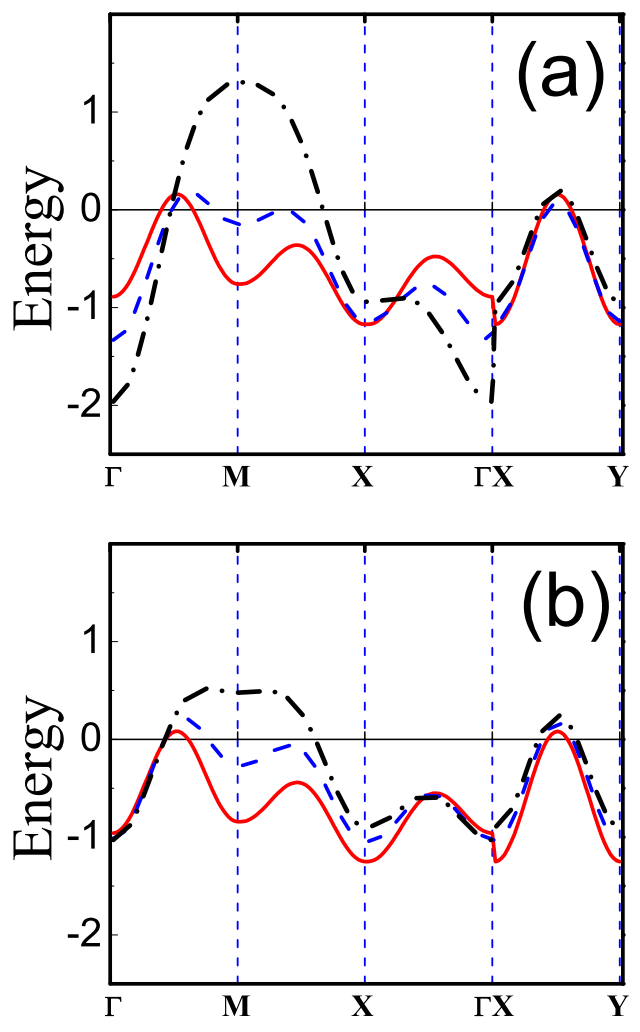

FIG. 2: (Color online) The same as in Figure 1 for $U=16$.

where $\widetilde{\varepsilon}(\mathbf{q})=\varepsilon_{2}(\mathbf{q}) / Z(\mathbf{q})$ is the renormalized energy. Whereas for the exchange interaction and CI there are no retardation effects and the pairing occurs for all electrons in the two-particle subband, the spin-fluctuation contributions is restricted to the range of energies $\pm \omega_{s}$ near the FS, as determined by the $\theta$-function.

To estimate various contributions in the gap equation (49) we consider a model $d$-wave gap function, $\varphi(\mathbf{k})=$ $(\Delta / 2) \eta(\mathbf{k})$ where $\eta(\mathbf{k})=\left(\cos k_{x}-\cos k_{y}\right)$. Then the gap equation can be written in the form (for detail see Ref. [18]):

$$
\begin{aligned}
& 1=\frac{1}{N} \sum_{\mathbf{q}} \frac{[1-b(\mathbf{q})]^{2}[\eta(\mathbf{q})]^{2}}{[Z(\mathbf{q})]^{2} 2 \widetilde{\varepsilon}(\mathbf{q})} \tanh \frac{\widetilde{\varepsilon}(\mathbf{q})}{2 T_{c}}\left\{J-V+\widehat{V}_{c f}\right. \\
& \left.+(1 / 4)|t(\mathbf{q})|^{2} \widehat{\chi}_{c f}-|t(\mathbf{q})|^{2} \widehat{\chi}_{s f} \theta\left(\omega_{s}-|\widetilde{\varepsilon}(\mathbf{q})|\right)\right\} .
\end{aligned}
$$

In this equation only $l=2$ components of the static susceptibility and CI give contributions

$$
\begin{aligned}
& \widehat{V}_{c f}=\frac{1}{N} \sum_{\mathbf{k}}|V(\mathbf{k})|^{2} \chi_{c f}(\mathbf{k}) \cos k_{x}, \\
& \widehat{\chi}_{c f}=\frac{1}{N} \sum_{\mathbf{k}} \chi_{c f}(\mathbf{k}) \cos k_{x}, \\
& \widehat{\chi}_{s f}=\frac{1}{N} \sum_{\mathbf{k}} \chi_{s f}(\mathbf{k}) \cos k_{x} .
\end{aligned}
$$

The contribution from the charge fluctuations $\hat{\chi}_{c f}$ (52) weakly depends on $U$ and $V$ and is very small:
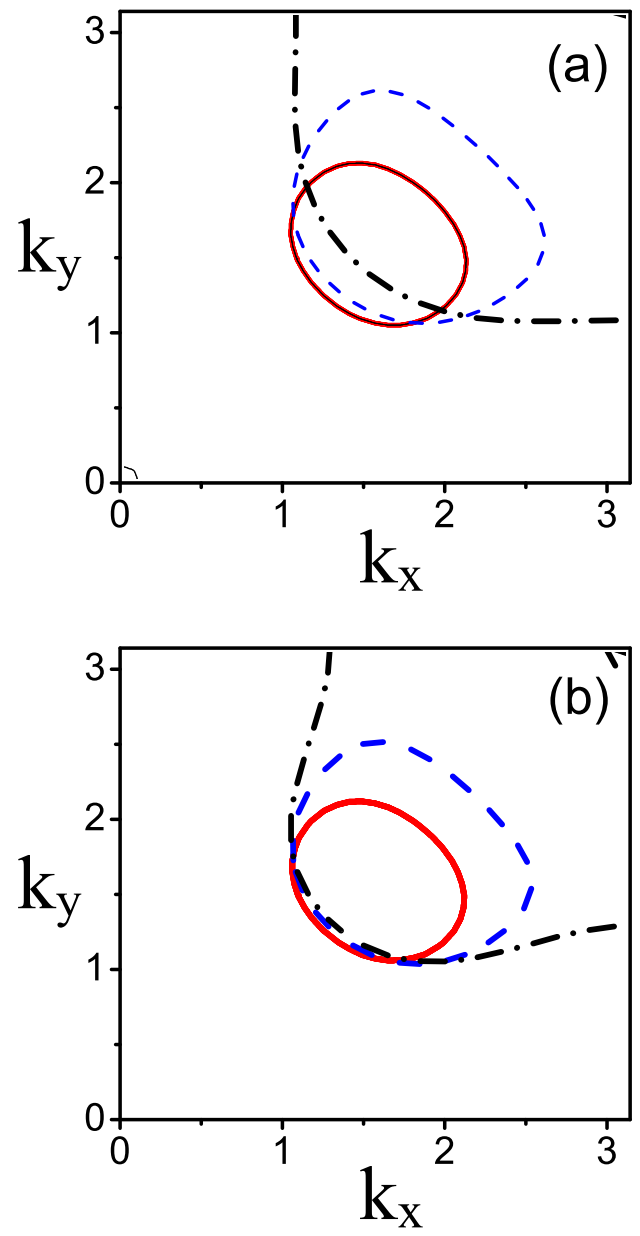

FIG. 3: (Color online) Fermi surface for (a) $V=0$ and (b) $V=2$ at $U=8$ in the quarter of the BZ in the GMFA at hole doping $\delta=0.05$ (red solid line), 0.10 (blue dashed line), and 0.25 (black dash-dotted line).

TABLE I: Charge-fluctuation contribution $\widehat{V}_{c f} / t$ for several values of the on-site CI $U$ and intersite CI $V$ for hole concentrations $\delta=0.10$.

\begin{tabular}{cccc}
\hline \hline$U$ & $V=1$ & $V=2$ & $V=3$ \\
\hline 8 & 0.10 & 0.29 & 0.53 \\
16 & 0.24 & 0.76 & 1.95 \\
32 & 0.43 & 1.47 & 1.71 \\
\hline \hline
\end{tabular}

$\widehat{\chi}_{c f} \sim 10^{-3}(1 / t)-10^{-2}(1 / t)$ for hole concentrations $\delta=0.05-0.10$, respectively. For the averaged over the BZ vertex $\overline{|t(\mathbf{q})|^{2}}=(1 / N) \sum_{\mathbf{q}}|t(\mathbf{q})|^{2} \simeq 4 t^{2}$ the contribution induced by the kinematic interaction is equal to $\overline{|t(\mathbf{q})|^{2}} \widehat{\chi}_{c f} \lesssim 0.04 t$ and can be neglected. The charge fluctuation contribution $\widehat{V}_{c f}$ (51) from the intersite CI (43) for the hole concentration $\delta=0.05$ is also small, $\widehat{V}_{c f} \lesssim 5 \cdot 10^{-2} t$ for $V \leq 2$ and increases up to $0.17 t$ for $V=4$. For larger hole concentration $\widehat{V}_{c f}$ increases as shown in Table \for $\delta=0.10$. However, $\widehat{V}_{c f}-V<0$ 

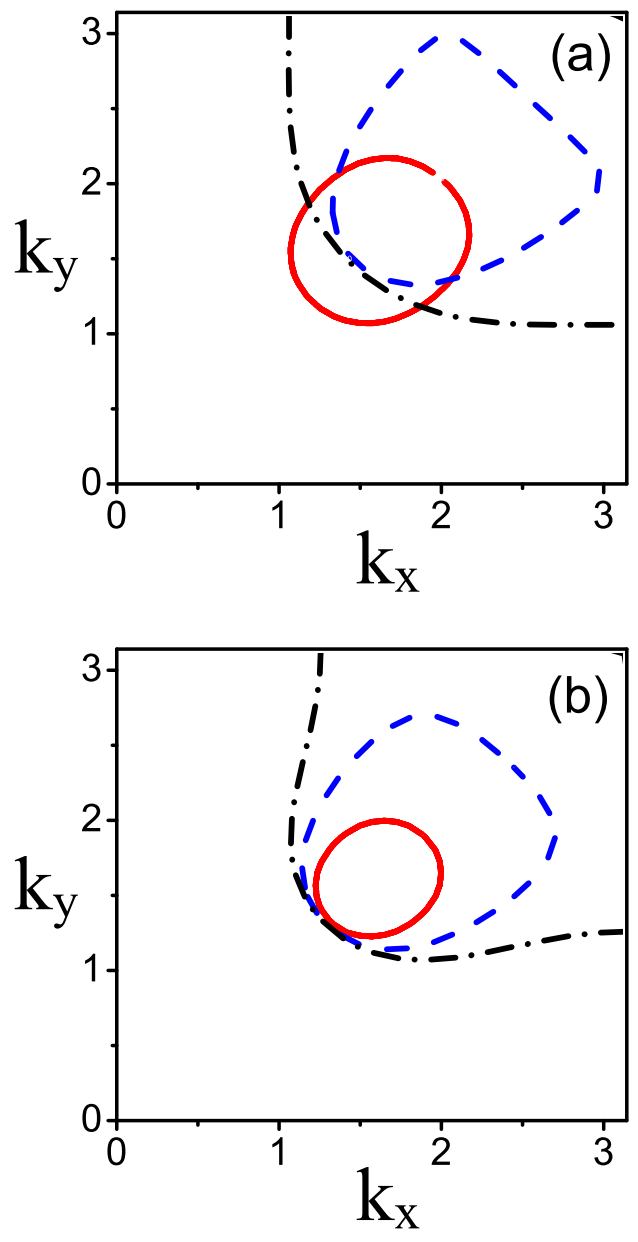

FIG. 4: (Color online) The same as in Figure 3 for $U=16$.

for all values of $U$ and $V$ and consequently, the $d$-wave pairing induced only by charge fluctuations cannot occur.

The spin-fluctuation contribution $\hat{\chi}_{s f}$ (53) is calculated for the model $\chi_{s f}(\mathbf{q})$ in Eq. (46). Since the spin susceptibility has a maximum at the $\mathrm{AF}$ wave vector $\mathbf{Q}=(\pi, \pi)$ the integral over $\mathbf{k}$ in (53) results in the negative value for $\hat{\chi}_{s f}$ which strongly depends on hole doping. Our previous calculations gave the following values: $-\widehat{\chi}_{s f} \cdot t \approx 1.3,1.0,0.6$ for hole concentrations $\delta=0.05,0.10,0.25$, respectively (see Ref. [18]). Using the averaged over $\mathrm{BZ}$ vertex $\overline{|t(\mathbf{q})|^{2}} \simeq 4 t^{2}$ we can estimate an effective spin-fluctuation coupling constant as $g_{s f} \simeq-4 t^{2} \hat{\chi}_{s f}=5.2,4.0,2.4$. Thus, the spinfluctuation contribution to the pairing in Eq. (50) with the coupling constant $g_{s f}=2-1 \mathrm{eV}$ for $\delta=0.05-0.25$ appears to be the largest.

Results of $T_{c}$ calculation using Eq. (50) are shown in Fig. 6 for (a) $U=8$ and (b) $U=16$ and $V=$ $0.0,0.5,1.0$, and 2. Similar doping dependence for $T_{c}$ is observed for $U=32$. The maximum $T_{c}$ at the optimal doping as a function of $U$ and $V$ is shown in Fig. 7. Increasing of the intersite Coulomb repulsion $V$ suppresses $T_{c}$ which becomes small only for high values of $V=2 t-3 t$
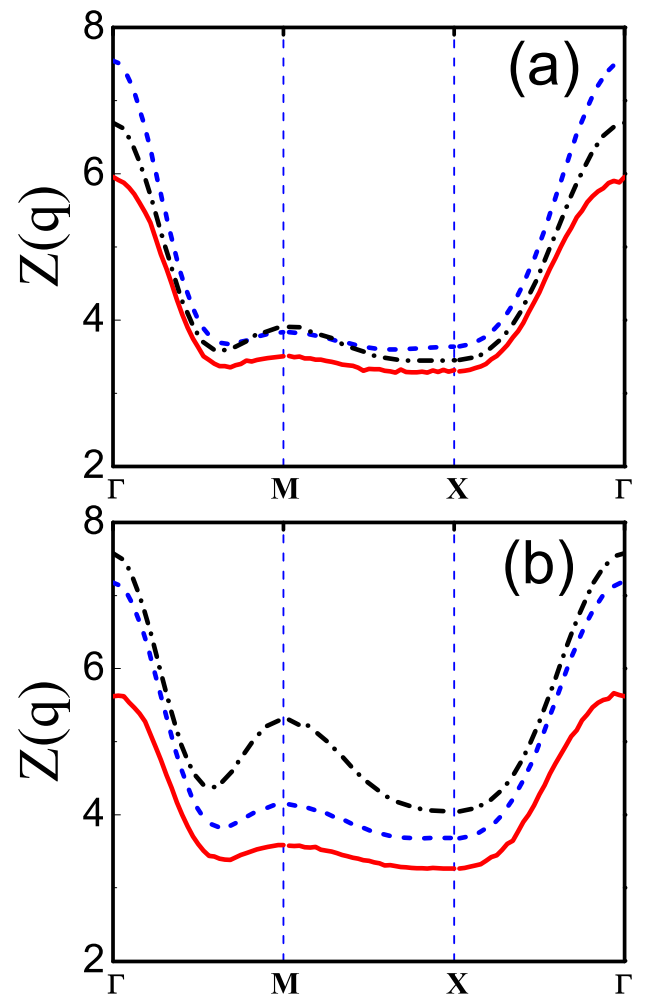

FIG. 5: (Color online) The renormalization parameter $Z(\mathbf{q})$ along the symmetry directions $\Gamma(0,0) \rightarrow M(\pi, \pi) \rightarrow$ $X(\pi, 0) \rightarrow \Gamma(0,0)$ at $\delta=0.10$ at $U=8$ (red solid line), $U=16$ (blue dashed line), and $U=32$ (black dash-dotted line) for (a) $V=0$ and (b) $V=1$.

comparable with the spin-fluctuation coupling $g_{s f}$ and much larger than the exchange interaction $J=0.4 t$. At the same time increasing of $U$ enhances $T_{c}$. This is due to narrowing of the electronic band as seen in Figs. 1, 2 and corresponding increase of the density of state.

To prove an important role of the spin-fluctuation interaction both in the normal state and in superconducting pairing we calculate the function $Z(\mathbf{q})$ (48) and $T_{c}$ for several values of the parameter $\omega_{s}$ for the static susceptibility in the model (46): $\omega_{s}=0.2,0.4,0.6$ and 1.0 for $U=8$. Figure 8 shows $T_{c}$ dependence on the parameter $\omega_{s}$ that determines the spin-fluctuation contribution $\widehat{\chi}_{s f}$ in Eq. (50) in two cases: for (a) $Z$ (q) given by Eq. (48) and (b) $Z(\mathbf{q})=1$. Since the spin-fluctuation interaction is determined by $\chi_{Q} \propto 1 / \omega_{s}$ (47) it increases with lowering of the cut-off frequency $\omega_{s}$. This results in increasing of the superconducting pairing contribution $\widehat{\chi}_{s f}$ but at the same time enhances the normal state renormalization $Z(\mathbf{q})$ as shown in Fig. 9. Therefore, in the case (a) $T_{c}$, roughly being proportional to $\widehat{\chi}_{s f} /[Z(\mathbf{q})]^{2}$, decreases due to suppression of the QP weight $1 / Z(\mathbf{q})$, while in the case (b) for $Z(\mathbf{q})=\mathbf{1}$ increasing of pairing strength results in $T_{c}$ increase. Note also, that $T_{c}$ in Fig. 8 (b) calculated in MFA with $Z(\mathbf{q})=1$ an order of magnitude larger than its value with a proper consideration of electronic spectrum renormalization. 

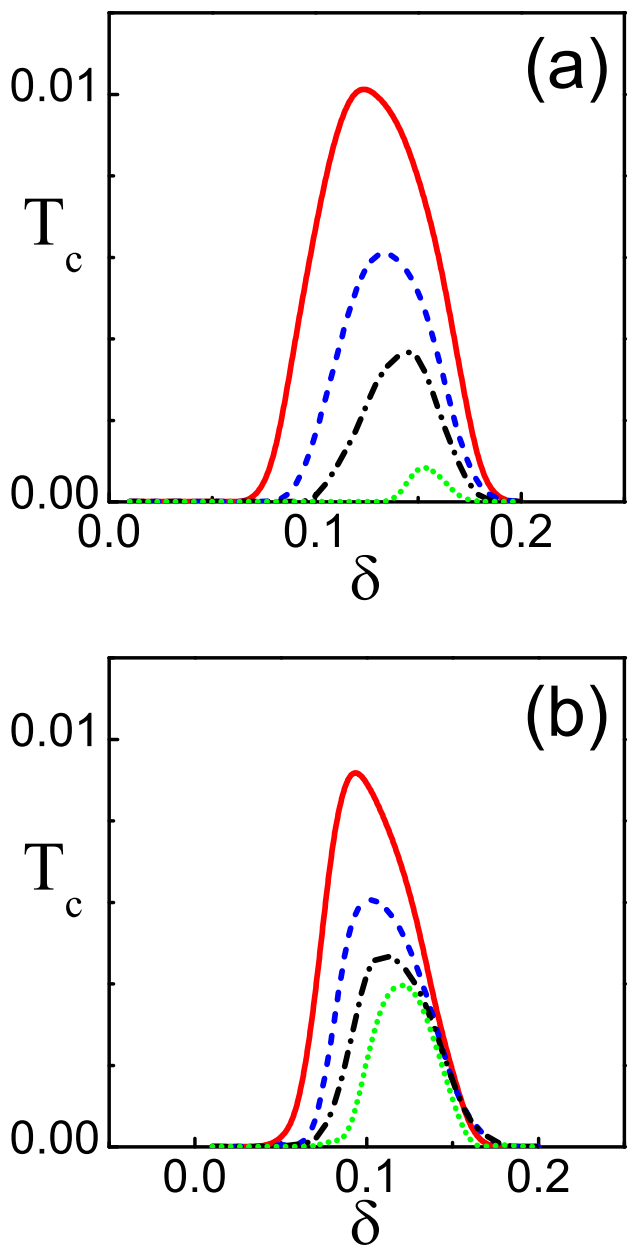

FIG. 6: (Color online) $T_{c}(\delta)$ for (a) $U=8$ and (b) for $U=$ 16 for $V=0.0$ (bold red line), $V=0.5$ (blue dashed line), $V=1.0$ (black dash-dotted line), and $V=2.0$ (green dotted line).

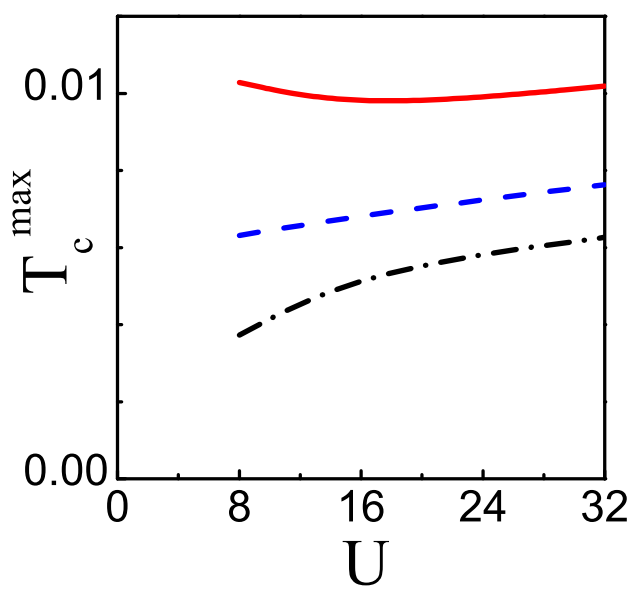

FIG. 7: (Color online) Maximum $T_{c}(\delta)$ as a function of $U$ for $V=0.0$ (bold red line), $V=0.5$ (blue dashed line), and $V=1.0$ (black dash-dotted line).
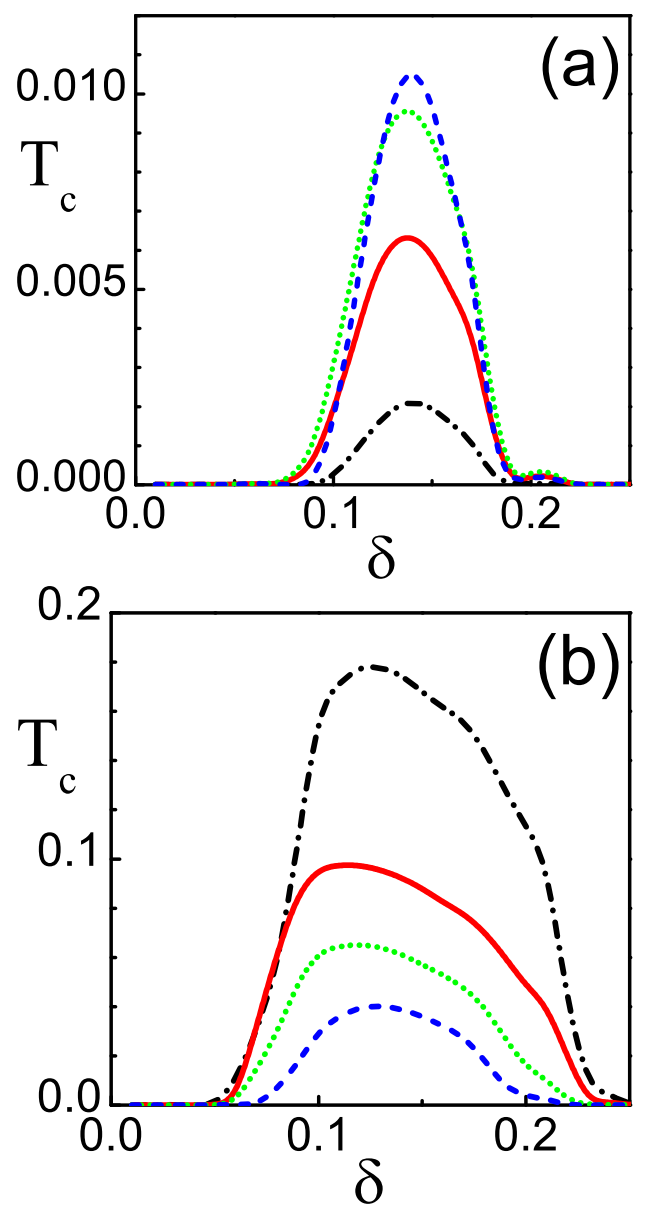

FIG. 8: (Color online) $T_{c}(\delta)$ dependence on spin-fluctuation contribution $\chi_{s f}$ in Eq. (50) for $\omega_{s}=0.2$ (black dash-dotted line), $\omega_{s}=0.4$ (bold red line),$\omega_{s}=0.6$ (green dotted line), and $\omega_{s}=1.0$ (blue dashed line) calculated for (a) finite $Z(\mathbf{q})$ and (b) $Z(\mathbf{q})=1$.

In the current approach one can also consider the $s$ wave pairing. For the extended $s$-wave gap function, $\varphi_{s}(\mathbf{k})=(\Delta / 2) \eta_{s}(\mathbf{k})$ where $\eta_{s}(\mathbf{k})=\left(\cos k_{x}+\cos k_{y}\right)$, a similar to (49) equation for $T_{c}$ can be derived. Solution of this equation reveals a finite and quite high $T_{c}$. However, $s$-wave pairing symmetry violates a kinematic restriction of no double occupancy for the Hubbard model in the two-subband regime. As was pointed out in Refs. [29, 30], the single-site correlation function should obey the condition

$$
\left\langle X_{i}^{\bar{\sigma} 2} X_{i}^{\sigma 2}\right\rangle=\frac{1}{N} \sum_{\mathbf{q}}\left\langle X_{-\mathbf{q}}^{\bar{\sigma} 2} X_{\mathbf{q}}^{\sigma 2}\right\rangle=0,
$$

caused by the multiplication rule for the Hubbard operators, $X_{i}^{\alpha \beta} X_{i}^{\gamma \delta}=\delta_{\beta \gamma} X_{i}^{\alpha \delta}$. In the QP approximation used in Eq. (49) we obtain the relation

$$
\left\langle X_{i}^{\bar{\sigma} 2} X_{i}^{\sigma 2}\right\rangle=\frac{1}{N} \sum_{\mathbf{q}} \frac{\varphi(\mathbf{q})}{[Z(\mathbf{q})]^{2} 2 \widetilde{\varepsilon}(\mathbf{q})} \tanh \frac{\widetilde{\varepsilon}(\mathbf{q})}{2 T_{c}}=0 .
$$

For the $d$-wave pairing $\varphi_{d}(\mathbf{q})=(\Delta / 2)\left(\cos q_{x}-\cos q_{y}\right)$ 


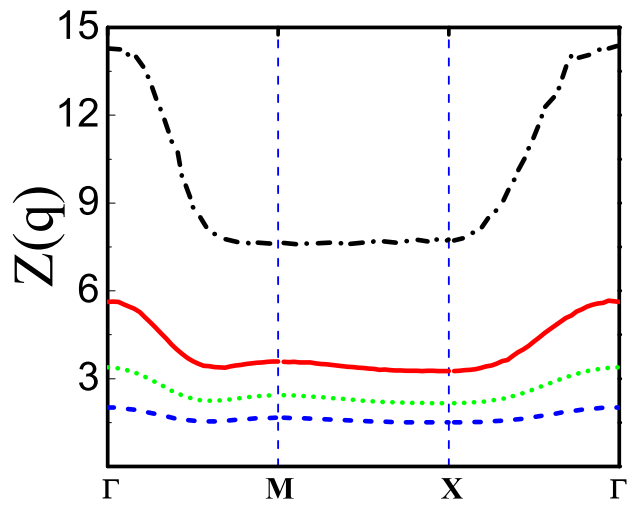

FIG. 9: (Color online) $Z(\mathbf{q})$ dependence on spin-fluctuation contribution $\chi_{s f}$ in Eq. (48) for $\omega_{s}=0.2$ (black dash-dotted line), $\omega_{s}=0.4$ (bold red line), $\omega_{s}=0.6$ (green dotted line), and $\omega_{s}=1.0$ (blue dashed line) at $\delta=0.10$.

this condition is fulfilled in the tetragonal phase for any doping (pairing in the orthorhombic pase is considered in Ref. [31]). For the $s$-wave pairing this condition is violated

$$
\frac{1}{N} \sum_{q_{x}, q_{y}} \frac{\cos q_{x}}{[Z(\mathbf{q})]^{2} 2 \widetilde{\varepsilon}(\mathbf{q})} \tanh \frac{\widetilde{\varepsilon}(\mathbf{q})}{2 T} \neq 0
$$

for an arbitrary doping except for a particular choice of the chemical potential when the contribution from the integral over $0 \leq q_{x} \leq \pi$ is compensated by the integral over $\pi \leq q_{x} \leq 2 \pi$. The same condition holds for the one-particle subband, $\left\langle X_{i}^{0 \bar{\sigma}} X_{i}^{0 \sigma}\right\rangle=0$. The obtained results can be derived for a general representation for the correlation function

$$
\left\langle X_{-\mathbf{q}}^{\bar{\sigma} 2} X_{\mathbf{q}}^{\sigma 2}\right\rangle=-\frac{1}{\pi Q_{2} N} \sum_{\mathbf{q}} \int_{-\infty}^{+\infty} \frac{d z}{\mathrm{e}^{z / T}+1} \operatorname{Im} F_{\sigma}^{22}(\mathbf{q}, z)
$$

since the symmetry of the anomalous $\operatorname{GF} F_{\sigma}^{22}(\mathbf{q}, z)$ is determined by the $s$ - or $d$-wave symmetry of the gap function. Therefore, we conclude that $s$-wave pairing is prohibited for the Hubbard model in the limit of strong correlations.

\section{Comparison with previous theoretical studies}

As discussed in Sec. I, the intersite Coulomb repulsion $V$ is detrimental for pairing induced by the on-site CI $U$ in the Hubbard model or higher-order contributions from $V$ in the weak correlation limit. Here we would like to comment on several studies of this problem in the strong correlation limit and to compare them with our analytical results for the $d$-wave pairing.

Following the original idea of Anderson [3], it is commonly believed that the exchange interaction $J=4 t^{2} / U$ induced by the interband hopping in the Hubbard model plays the major role in the $d$-wave superconducting pairing. Since the excitation energy of electrons in the interband hopping $U$ is much larger than their intraband kinetic energy $W$ the exchange pairing has no retardation effects contrary to the electron-phonon pairing where large Bogoliubov-Tolmachev logarithm [27] diminishes the Coulomb repulsion $V \rightarrow V /\left[1+\rho_{c} \ln \left(\mu / \omega_{p h}\right)\right]$ where $\rho_{c}=N(0) V$ and $\omega_{p h}$ is the phonon energy. Consequently, without the retardation effects the Coulomb repulsion $V$ should destroy the exchange pairing for $V>J$.

To get over this problem, in Ref. [16] it was suggested that in the limit of strong correlation the intersite Coulomb repulsion $V$ decreases the interband excitation energy which results in enhancement of the exchange interaction, $\widetilde{J}(V)=4 t^{2} /(U-V)$, as was found from cluster calculations. If we consider pairing induced only by the exchange interaction $\widetilde{J}(V)$ and take into account the Coulomb repulsion $V$ then the condition $\widetilde{J}(V)-V>0$ should be fulfilled for existence of pairing. The condition is satisfied for $0<V<V_{1}$ where $V_{1}=(U / 2)\left[1-\sqrt{1-(4 t / U)^{2}}\right]$ for $0 \leq V<U$. For $U>4 t$ we have $V_{1} \ll U$ as, e.g., for $U=8, V_{1}=0.067 U$ and for $U=32, V_{1}=0.004 U$. Therefore, we see that the pure exchange superconducting pairing can occur in the region of weak Coulomb repulsion. Contrary to this, in Ref. [16] using the cellular dynamical mean-Field theory (CDMFT) [14] the $d$-wave pairing was found in the region of strong coupling up to $V \lesssim U / 2$ (as, e.g., shown in Fig. 3, $V \leqslant 3 t(8 t)$ for $U=8 t(16 t)$, respectively). At the same time, in the limit of weak correlations $U=4 t$ the pairing is suppressed at the smaller value of $V \sim 1.5 t$. Thus, we believe that "Resilience of $d$-wave superconductivity to nearest-neighbor repulsion" is not due to renormalization of the exchange interaction $\widetilde{J}(V)$ but due to another mechanism of pairing not explicitly seen in the CDMFT calculations. As we have shown in the strong correlation limit in the two-subband regime the emerging kinematic interaction is responsible for the spin-fluctuation pairing at large values of $V$, up to $V \lesssim 4 t$.

Our conclusion about importance of the kinematic mechanism of pairing is supported by the studies in Ref. [15]. Using the variational Monte Carlo technique the superconducting $d$-wave gap was calculated for the extended Hubbard model with a weak exchange interaction $J=0.2 t$ and a repulsion $V \leq 3 t$ in a broad range of $0 \leq U \leq 32$. It was found that the gap decreases with increasing $V$ at all $U$ and can be suppressed for $V>J$ for small $U$. But for large $U \gtrsim U_{c} \sim 6 t$ the gap becomes robust and exists up to large values of $V \sim 10 \mathrm{~J}=2 \mathrm{t}$ which was explained by effective enhancement of $J$ as in Ref. [16]. At the same time, the gap does not show notable variation with $U$ for large $U=10-30$ though it should depend on the conventional exchange interaction in the Hubbard model $J=4 t^{2} / U$ (or $J=4 t^{2} /(U-V)$ ). We can suggest another explanation of these results by pointing out that at large $U \gtrsim U_{c}$ concomitant decrease 
of the bandwidth (as shown in Fig. 3 b) in Ref. [15]) results in the splitting of the Hubbard band into the upper and lower subbands and the emerging kinematic interaction induces the $d$-wave pairing in one Hubbard subband. In that case the second subband for large $U$ gives a small contribution which results in $U$-independent pairing. It can be suppressed by the repulsion $V$ only larger than the kinematic interaction, $V \gtrsim 4 t$.

In Ref. [17 the extended Hubbard model is considered in the weak or intermediate correlation limits as in Ref. [6] and in the strong correlation limit within the slave-boson representation in the mean-field approximation (MFA). In the strong correlation limit a small value of $V=J$ was found which suppresses the $d$-wave superconducting gap. However, in the MFA the kinetic energy term described by the projected electron operators, $t \hat{c}_{i \sigma}^{\dagger} \hat{c}_{j \sigma}=t c_{i \sigma}^{\dagger}\left(1-n_{i-\sigma}\right) c_{j \sigma}\left(1-n_{j-\sigma}\right) \equiv t X_{i}^{\sigma 0} X_{j}^{0 \sigma}$, is approximated by the conventional fermion (spinon) operators, $t \delta f_{i \sigma}^{\dagger} f_{j \sigma}$ and the most important contribution from the kinematic interaction is lost in the resulting BCS-type gap equation (13) in Ref. [17]. As shown in our equation for the gap (50) the kinematic interaction given by $\widehat{\chi}_{s f}$ (53) provides strong spin-fluctuation pairing and high $T_{c}$.

To analyze the pairing mechanisms in the limit of strong correlations analytical methods should be used. A complicated dynamics of projected electron operators can be rigorously taken into account using the HO technique. The algebra of the HOs preserves rigorously restriction of no double occupancy of quantum states which is violated in the commonly used MFA in the slaveparticle theory. As discussed in Sec. II the commutation relations for the HOs results in the kinematic interaction which is responsible for strong spin-fluctuation electron interaction. The superconducting pairing induced by the kinematic interaction for the HOs was first proposed by Zaitsev and Ivanov [28] who studied the twoparticle vertex equation by applying the diagram technique for HOs. The momentum-independent $s$-wave superconducting gap was found which, however, violates the HO kinematics as was shown in Refs. [29, 30] (see Eqs. (54) - (56) ). Since the intersite Coulomb repulsion $V>J$ destroys the superconductivity induced by the AF exchange interaction, the spin-fluctuation pairing in the second order of the kinematic interaction beyond the GMFA should be taken into account as discussed in detail in Sec. IVB and for the $t-J$ model was considered in
Refs. [32, 33].

\section{CONCLUSION}

In the paper we have studied effects of the strong intersite Coulomb repulsion $V$ on the $d$-wave superconducting pairing within the extended Hubbard model (1) in the limit of strong electron correlations, $U \gg t$. Using the Mori-type projection technique we obtained a selfconsistent system of equations for normal and anomalous (pair) GFs and for the self-energy calculated in the SCBA.

It was found that the kinematic spin-fluctuation interaction $g_{s f}$ induced by electron hopping in one Hubbard subband is much stronger than the conventional exchange interaction $J$ resulting from the interband hopping. Consequently, the $d$-wave pairing can be suppressed only for large values of $V>g_{s f}$ where $g_{s f}$ is of the order of kinetic energy $g_{s f} \sim W \approx 4 t$. Since in the cuprates the Coulomb repulsion $V$ is of the same order as the exchange interaction, $V \gtrsim J \sim 0.4 t$, the kinematic spinfluctuation pairing mechanism plays the major role in achieving high-temperature superconductivity. It is also shown that the kinematic spin-fluctuation interaction results in a strong renormalization of electronic spectra.

It is important to point out that the superconducting pairing induced by the AF exchange interaction and the spin-fluctuation kinematic interaction is characteristic for systems with strong electron correlations. These mechanisms of superconducting pairing are absent in the fermionic models and are generic for cuprates. Therefore, we believe that the spin-fluctuation kinematic mechanism of superconducting pairing in the Hubbard model in the limit of strong correlations is the relevant mechanism of high-temperature superconductivity in the copper-oxide materials.

\section{Acknowledgments}

The authors would like to thank A.S. Alexandrov, V.V. Kabanov, A.-M. S. Tremblay and M.Yu. Kagan for valuable discussions. Partial financial support by the Heisenberg-Landau Program of JINR is acknowledged.
[1] Handbook of High-Temperature Superconductivity. Theory and Experiment, edited by J. R. Schrieffer and J. S. Brooks (Springer-Verlag, New York, 2007).

[2] N. M. Plakida, High-Temperature Cuprate Superconductors (Springer Series in Solid-State Sciences, Vol. 166, Springer-Verlag, Berlin, 2010), Chap. 7.

[3] P. W. Anderson, Science 235, 1196 (1987); P. W. Anderson, The theory of superconductivity in the high-Tc cuprates (Princeton University Press, Princeton, 1997).

[4] J. Hubbard, Proc. Roy. Soc. (London) A, 276, (1963) 238.

[5] A. S. Alexandrov and V. V. Kabanov, Phys. Rev. Lett. 106, 136403 (2011).

[6] S. Raghu, E. Berg, A. V. Chubukov, and S. A. Kivelson Phys. Rev. B 85, 024516 (2012).

[7] W. Kohn and J. M. Luttinger, Phys. Rev. Lett. 15, 524 
(1965).

[8] M. Yu. Kagan, D. V. Efremov, M. S. Marienko, and V. S. Val'kov. JETP Lett. 93725 (2011).

[9] D. V. Efremov, M. S. Marenko, M. A. Baranov, and M. Yu. Kagan, J. Exp. Theor. Phys. 90, 861 (2000).

[10] M. Yu. Kagan, V. V. Val'kov, V. A. Mitskan, and M. M. Korovushkin, J. Exp. Theor. Phys. 144, 837 (2013).

[11] E. Dagotto, Rev. Mod. Phys. 66, 763 (1994).

[12] N. Bulut, Advances in Physics 51, 1587 (2002).

[13] D. J. Scalapino, Numerical studies of the 2D Hubbard model, in Ref. 1], pp. 495-526.

[14] D. Sénéchal, Cluster dynamical mean field theory, in Theoretical methods for Strongly Correlated Systems, edited by A. Avella and F. Mancini ( Springer Series in SolidState Sciences, Vol. 171, Springer- Verlag, Berlin, 2012), Chap. 11.

[15] E. Plekhanov, S. Sorella, and M. Fabrizio, Phys. Rev. Lett. 90, 187004 (2003).

[16] D. Sénéchal, A. Day, V. Bouliane, and A.-M. S. Tremblay, arXiv:1212.4503 [cond-mat.supr-con].

[17] S. Raghu, R. Thomale, and T. H. Geballe, Phys. Rev. B 86, 094506 (2012).

[18] N. M. Plakida and V. S. Oudovenko, Eur. Phys. J. B 86, 115 (2013).

[19] J. Hubbard, Proc. Roy. Soc. A (London) 285, (1965) 542.

[20] H. Mori, Prog. Theor. Phys. 34, 399 (1965).

[21] D. N. Zubarev, Usp. Fiz. Nauk 71, 71 (1960); (Sov. Phys. Usp. 3, 320 (1960)); Nonequilibrium Statical Thermodynamics (Consultant Bureau, New-York, 1974).

[22] N. M. Plakida and V. S. Oudovenko, JETP 104, 230 (2007)
[23] N. M. Plakida, L. Anton, S. Adam, and Gh. Adam, Zh. Exp.Theor. Fyz. 124, 367 (2003), (JETP 97, 331 (2003)).

[24] G. M. Eliashberg, Zh. Eksp. Teor. Fiz. 38, 966 (1960); ibid 39, 1437 (1960) (Soviet Phys. JETP 11, 696 (1960); ibid 12, 1000 (1960)).

[25] J. Jaklič and P. Prelovśek, Phys. Rev. Lett. 74, 3411 (1995); ibid. 75, 1340 (1995).

[26] A. A. Vladimirov, D. Ihle, and N. M. Plakida, Phys. Rev. B 80, 104425 (2009).

[27] N. N. Bogoliubov, V. V. Tolmachev, and D. V. Shirkov, New method in the theory of superconductivity, (Publ. Dept. USSR Acad. of Science, Moscow, 1958; Consultants Bureau, Chapman and Hall, New York - London, 1959, Vol. YII).

[28] R. O. Zaitsev, and V. A. Ivanov, Soviet Phys. Solid State 29, 2554 (1987), Ibid. 29, 3111 (1987), Int. J. Mod. Phys. B 5, 153 (1988)

[29] N. M. Plakida, V. Yu. Yushankhai, and I. V. Stasyuk, Physica C 160, 80 (1989).

[30] V. Yu. Yushankhai, N. M. Plakida, and P. Kalinay, Physica C 174, 401 (1991).

[31] N. M. Plakida and V. S. Oudovenko, Physica C, 341348, 289 (2000); in Proceedings of the NATO ARW on open problems in strongly correlated systems, edited by J.Bonca, P. Prelovsek, A. Ramsak and S. Sarkar (Kluewer Academic Publs., 2001) p. 111-116.

[32] N. M. Plakida and V. S. Oudovenko, Phys. Rev. B 59 11949 (1999).

[33] P. Prelovšek and A. Ramšak, Phys. Rev. B 72, 012510 (2005). 